\title{
Fisheries and Limnology of Two Reservoirs in Northern Ghana
}

\author{
T. Quarcoopome*, F. Y. K. Amevenku and O. D. Ansa-Asare \\ CSIR-Water Research Institute, P. O. Box AH 38, Achimota, Ghana, West Africa \\ *Corresponding author; E-mail: qpome@yahoo.com
}

\begin{abstract}
The fisheries and limnology of Bontanga and Libga reservoirs in northern Ghana were studied with the objective of generating information to support the development, management and sustainable use of their fisheries to enhance the socio-economic status of riparian communities. Using gill nets of various mesh sizes, fishes were sampled monthly and individually identified, weighed and measured for standard and total lengths. Water samples were taken monthly and analysed for physico-chemical parameters. In the Bontanga Reservoir, 21 species belonging to 18 genera and 10 families were encountered. Four families, namely Clariidae, Cichlidae, Cyprinidae and Clarotidae constituted almost $83 \%$ of the fishery while Clarias anguillaris, Auchenoglanis occidentalis, Barbus macrops and Sarotherodon galileus were the major species. In the Libga Reservoir, 36 species, representing 27 genera and 15 families, were obtained. Two families, namely Mochokidae and Centropomidae constituted almost $67 \%$ of the fishery resources while Synodontis gambiensis and the Nile perch, Lates niloticus, were the dominant species. The fish diversity of the Bontanga Reservoir was lower than that of the Libga reservoir. Carnivorous fishes were dominant in Bontanga Reservoir while fishes of forage feeding habits were the major feeding group in Libga Reservoir. The forage-carnivore ratios of 1.60 and 2.12 for Bontanga and Libga reservoirs, respectively, suggest a suitable ecological balance between carnivorous fishes and their prey populations. The estimated potential fish yield per year for the Bontanga Reservoir (67 tons) was higher than that of the Libga Reservoir (4.7 tons) mainly due to its larger size although the productivity per hectare for Libga Reservoir was slightly higher. Both reservoirs were well oxygenated all year-round with relatively high oxygen demanding substances and slightly acidic from June to October. The Bontanga Reservoir was characterized by a strikingly high ionic content. The nutrient level and the mean productivity of the Bontanga Reservoir were slightly lower than those of the Libga Reservoir.
\end{abstract}

\section{Introduction}

Water is increasingly becoming scarce because of extensive desertification and increasing demands by growing and developing human populations. In the northern part of Ghana where long dry seasons and a single unreliable rainy season prevail, the construction of dugouts and small reservoirs or dams has been implemented over the past years to provide reliable water supplies to the local rural communities. These surface water collection systems are now widespread throughout the Northern Region where they have become a vital part of the infrastructure of the local communities. Although the relevance of these impoundments in terms of fish production was not seriously considered at the time of construction, many of them now support thriving fisheries. The utilisation of impoundments for fish production, besides other primary uses, has become common practice in many countries, especially industrialised ones, where a number of impoundments are managed for the production of annual harvestable fish crops (Byrd \& Moss, 1957; Davies, 1973). Although fish forms an important component of the diet of many people in Ghana, farther away from the coast of Ghana, fish becomes relatively scarce and expensive. This situation is worse within the rural settlements of the Northern Region of Ghana.

Given requisite information and adequate management, reservoirs in Ghana have the potential to complement fisheries in larger inland water bodies, thus, increasing the availability of fish. This study, therefore, was aimed at generating information on the state of the fish fauna and limnology of the two reservoirs following 10 and 25 years, respectively, of their existence to assist in the development, management and sustainable use of the fisheries of the reservoirs to enhance the socio-economic status of their respective riparian communities.

West African Journal of Applied Ecology, Vol. 12, 2008 
Study areas

\section{Materials and methods}

The Bontanga Reservoir (Fig. 1), located in the Tolon Kumbugu District of the Northern Region, was impounded in 1986. The reservoir has a surface area of 770 ha at maximum height (IDA, 1986) and is the largest in the Northern Region. The Bontanga Reservoir lies between latitudes $9^{\circ}$ $25^{\prime}$ and $9^{\circ} 45^{\prime} \mathrm{N}$ and longitudes $0^{\circ} 45^{\prime}$ and $1^{\circ} 15^{\prime} \mathrm{W}$. Although it was established for irrigation purposes, the reservoir currently supports a thriving fishery which offers enormous opportunities for increasing freshwater fish production in the Region. Information on the limnology and fisheries of the reservoir are scanty. Notable among the few reports available, however, are the survey of the fisheries (Datua, 1989), an assessment of the potential of the reservoir for aquaculture (Mayachi, 1991) and survey of aquatic weeds (deGraft-Johnson, 1995). 


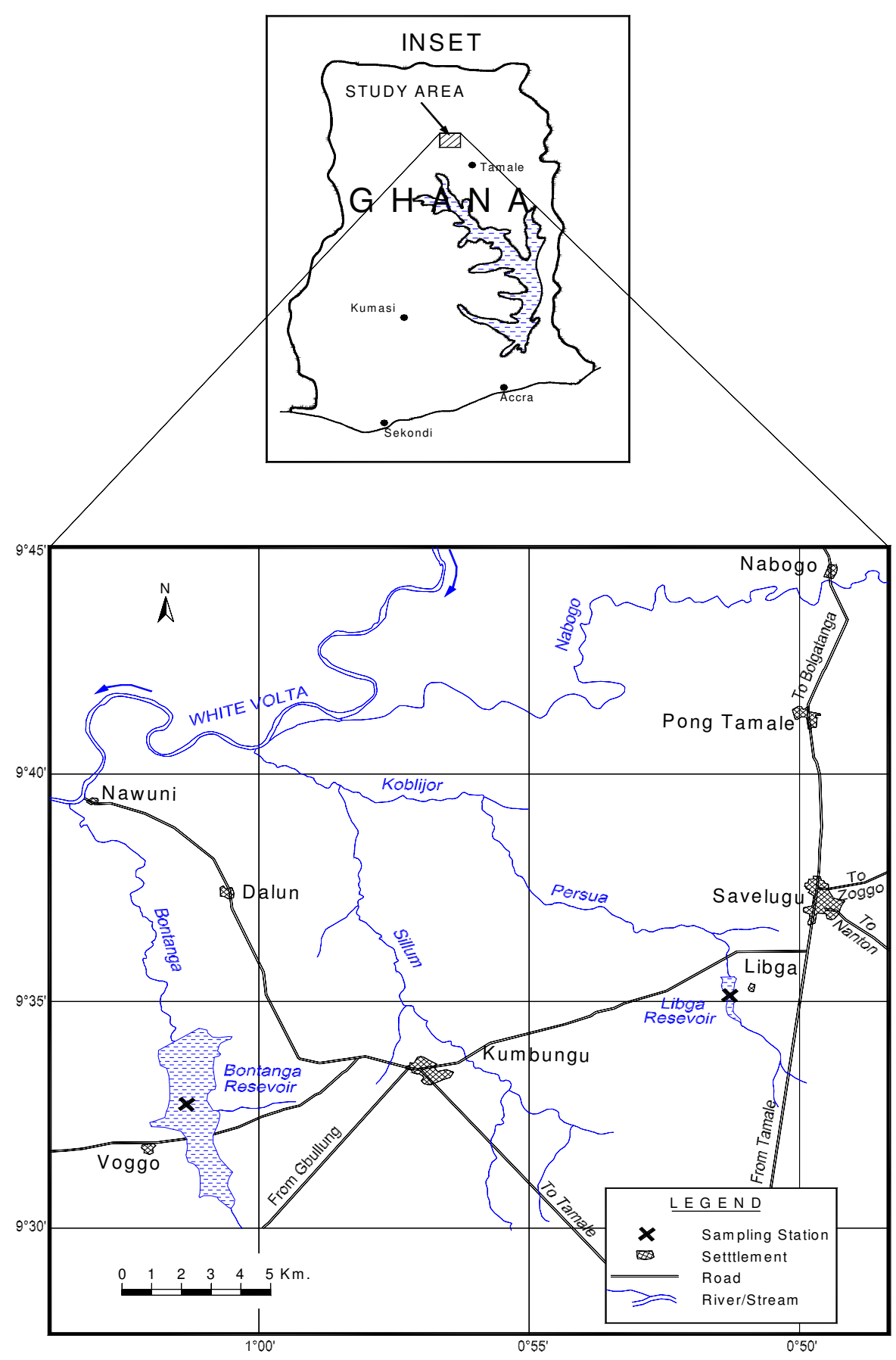

Fig. 1. Map of Ghana showing Bontanga and Libga reservoirs (sampling stations) in northern Ghana

The Libga Reservoir ( $9^{\circ} 35^{\prime} \mathrm{N}, 0^{\circ} 51^{\prime} \mathrm{W}$ ) (Fig. 1) in the Savelugu-Nanton District has a surface area of 48 ha at maximum height. It was impounded in 1970 but the wall collapsed in the same year, flooding the downstream areas (Wayo, personal com-munication) and subsequent rehabilitation was completed in 1971. Although it was constructed for irrigated agriculture, it is currently being utilised for a variety of domestic purposes. Besides these benefits, the Libga Reservoir is of immense economic importance to the peasants who have developed survival strategies based on access to both fishing and farming. 


\section{Sampling and data analysis}

Fish and water samples were taken monthly for a period of 1 year (July 1995-June 1996). Seasons were defined as rainy (July-September) and dry (October-June).

\section{Fish sampling and data analysis}

Fish were sampled with a set of multifilament gill nets of laterally stretched mesh sizes that ranged between 12.5 and $40.0 \mathrm{~mm}$, and a set of monofilament gill nets of large meshes ranging from 50.0 to $212.5 \mathrm{~mm}$. All nets were set in the late afternoon between 1600 and $1800 \mathrm{GMT}$ and retrieved the next morning between 0600 and 0800 GMT. Two nights of fishing were conducted each month. The fish obtained were identified individually with the assistance of keys by Lévêque et al. (1992), sexed, measured and weighed. Specimens were measured for standard length (SL) and total length (TL) to the nearest $0.1 \mathrm{~mm}$, and the body weight (BW) taken to the nearest $0.1 \mathrm{~g}$. The following parameters were computed for fish analysis.

Fish species composition and diversity. Fish species composition of both reservoirs was estimated from the checklist of fishes obtained from identification of monthly samples. A number of indices were used to describe and compare the diversity of the fish communities in the two reservoirs. These were (i) Margalef's Index (D) for species richness (Margalef, 1968).

$\mathrm{D}=(\mathrm{S}-1) / \mathrm{Ln} \mathrm{N}$

where $\mathrm{S}=$ number of species and $\mathrm{N}=$ number of individuals. (ii) The Shannon-Wiener's Index $\left(H^{\prime}\right)$ of species diversity (Shannon \& Wiener, 1963);

$$
\mathrm{H}^{\prime}=-\sum \mathrm{P}_{\mathrm{i}} \operatorname{Ln} \mathrm{P}_{\mathrm{i}}
$$

where $\mathrm{P}_{\mathrm{i}}$ is the proportion of the total number of individuals occurring in species i. (iii) Pielou's Index (J) for species evenness (Pielou, 1969);

$$
\mathrm{J}=\mathrm{H}^{\prime} / \mathrm{Ln} \mathrm{S}
$$

where $\mathrm{H}^{\prime}$ is the species diversity index and $\mathrm{S}$ is the number of species.

The ecological balance of the community was estimated by the ratio of forage fishes to carnivore fishes (i.e. forage-carnivore or F/C ratio) in terms of weight. Catch per unit effort (CPUE) of all fish caught by the small-sized meshes (i.e. $12.5 \mathrm{~mm}, 15.0 \mathrm{~mm}$ and $17.5 \mathrm{~mm}$ ) were computed using Lévêque et al. (1988).

CPUE $=\mathrm{W} * 100 / \mathrm{L} * \mathrm{n} * \mathrm{~d}$

where $\mathrm{W}$ is the weight of fish caught in grammes $(\mathrm{g})$, $\mathrm{L}$ is the length of net immersed in metres $(\mathrm{m}), \mathrm{n}$ is the number of nights of fishing, and $\mathrm{d}$ is the depth of the fishing net in metres $(\mathrm{m})$. Furthermore, the relationship between the monthly CPUE of all small mesh-sized nets together and monthly water levels (in metres) was investigated for each reservoir.

Growth parameters of the most frequently occurring species in the two reservoirs were estimated from length frequency data using FISAT microcomputer software (Gayanilo et al., 1996). The growth parameters estimated were maximum attainable (asymptotic) length ( $\mathrm{L} \infty$ ), growth constant $(\mathrm{K})$ and growth performance index $(\varnothing)$. The six fish species identified as frequently occurring constituted the most important species in the two reservoirs.

Potential fish yield estimates were obtained using abiotic variables based on the chemical composition of the reservoirs and the relationship:

$$
\mathrm{Y}=23.281 \mathrm{MEI}^{0.447} \text { (Marshall, 1984) }
$$

where $\mathrm{Y}$ is the potential fish yield in $\mathrm{kg} \mathrm{ha}^{-1}$, MEI is morphoedaphic index, which is given in $\mu \mathrm{m} / \mathrm{hos}$ and is estimated by dividing the mean conductivity by the mean depth (Ryder et al., 1974).

Water sampling and analyses of physico-chemical parameters 
Water samples were collected into 500-ml plastic bottles. Temperature and $p \mathrm{H}$ were determined in situ using a mercury-in-glass thermometer and a portable $p \mathrm{H}$ meter, respectively. For dissolved oxygen (DO) determination, separate samples were collected in plain glass bottles and the azide modification of the Winkler's method used. Samples for biochemical oxygen demand (BOD) were collected in dark glass bottles and incubated at $20{ }^{\circ} \mathrm{C}$ for 5 days after which the remaining DO was determined. All nutrients were analysed within 0.1-0.9\% relative standard deviation (RSD) using colorimetric methods. All the methods used for analyses of the parameters were essentially those in the Standard Methods for the Examination of Water and Waste-water (APHA-AWWA-WPCF, 1998).

Fish species composition

\section{Results}

The checklist of fishes (Table 1) in the Bontanga Reservoir indicated 21 species belonging to 18 genera and 10 families while that of the Libga Reservoir indicated 36 species made up of 27 genera and 15 families. The percentage fish species composition in terms of weight (Table 2) for the Bontanga Reservoir indicated that the dominant species were Clarias anguillaris $(36.22 \%)$, Auchenoglanis occidentalis (13.43\%), Barbus macrops (12.74\%) and Sarotherodon galileus (12.48\%). Ten species had percentage weight compositions ranging between 1.00 and $5.00 \%$. These were L. coubie (1.33\%), P. bovei (1.83\%), S. gambiensis (1.99\%), O. niloticus (2.03\%), M. senegalensis (2.14\%), H. fasciatus (2.29\%), T. zillii (2.35\%), B. nurse (2.92\%), H. niloticus (3.04\%) and S. intermedius (4.93\%). Fish species with percentage weight composition less than $1.00 \%$ included $P$. isidori (0.001), L. niloticus (0.003), B. leuciscus (0.004), P. senegalus $(0.01 \%)$, S. eupterus $(0.03 \%)$, P. bane $(0.03 \%)$ and B. brachyiustus $(0.21 \%)$.

TABLE 1

Checklist and estimated diversity indices of fish species in Bontanga and Libga reservoirs in northern Ghana (July 1995-June 1996)

Family/Species

Anabantidae

Ctenopoma kingslaye

Clarotidae

Centropomidae

Lates niloticus

Characidae

Alestes baremoze

Brycinus leuciscus

Brycinus nurse

Hydrocynus forskalii

Cichlidae

Hemichromis bimaculatus

Hemichromis fasciatus

Oreochromis niloticus

Sarotherodon galileus

Tilapia zillii

Citharinidae

Citharinus citharus
Common name

Climbing perch

Auchenoglanis occidentalis Claroteids

Nile perch

Silversides

Characins

Characins

Tiger fish

Tilapias

Tilapias

Nile tilapia

White tilapia

Redbelly tilapia

Moonfish
Libga

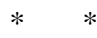

$*$

$*$

$*$

$*$

$*$

$*$

West African Journal of Applied Ecology, Vol. 12, 2008 
Clariidae

Clarias anguillaris

Mudfish

$=$ Present.

TABLE 1 cont'd

Family/Species

Cyprinidae

Barbus macrops

Labeo coubie

Labeo senegalensis

Leptocypris niloticus

Distichodontidae

Distichodus rostratus

Malapteruridae

Malapterurus electricus

Mochokidae

Synodontis eupterus

Synodontis gambiensis

Synodontis ocelifer

Synodontis velifer

Mormyridae

Brienomyrus brachyistus

Hyperopsis bebe

Marcusenus senegalensis

Mormyrus macrophthalmus

Mormyrus rume

Petrocephalus bane

Petrocephalus bovei

Pollimyrus isidori

Osteoglossidae

Heterotis niloticus

Polypteridae

Polypterus senegalensis

Schilbeidae

Schilbe intermedius

Schilbe mystus
Common Name

Blackstripe barb

African carp

African carp

Distichodonts

Electric fish

Lizard fish

Lizard fish

Lizard fish

Lizard fish

Elephant nose fishes

Elephant nose fishes

Mormyrid

Elephant fish

Bony tongue

Sail fins

Butter fish

Butter fish
Bontanga

Libga

-

$$
\text { - } \quad=\text { Present }
$$

TABLE 2

Percentage representation of fish species by weight in Bontanga and Libga reservoirs in northern Ghana (July 1995 June 1996)

\begin{tabular}{|c|c|c|c|c|}
\hline \multirow[b]{2}{*}{ Species } & \multicolumn{2}{|c|}{ Bontanga } & \multicolumn{2}{|c|}{ Libga } \\
\hline & Weight $(g)$ & Percentage (\%) & Weight $(g)$ & Percentage (\%) \\
\hline Alestes baremoze & ND & ND & 3000 & 0.87 \\
\hline
\end{tabular}

West African Journal of Applied Ecology, Vol. 12, 2008 


\begin{tabular}{|c|c|c|c|c|}
\hline Auchenoglanis occidentalis & 82681 & 13.43 & 18290 & 5.32 \\
\hline Barbus macrops & 78476 & 12.74 & 1020 & 0.30 \\
\hline Brienomyrus brachyistus & 1272 & 0.21 & 345 & 0.10 \\
\hline Brycinus leuciscus & 3 & 0.00048 & 552 & 0.1 \\
\hline Brycinus nurse & 17979 & 2.92 & 20536 & 5.98 \\
\hline Citharinus citharus & ND & ND & 859 & 0.25 \\
\hline Clarias anguillaris & 223069 & 36.22 & 17037 & 4.96 \\
\hline Ctenopoma kingslaye & ND & ND & 33 & 0.01 \\
\hline Distichodus rostratus & ND & ND & 1719 & 0.50 \\
\hline Hemichromis bimaculatus & ND & ND & 33 & 0.01 \\
\hline Hemichromis fasciatus & 14093 & 2.29 & 27 & 0.01 \\
\hline Heterotis niloticus & 18714 & 3.04 & 7321 & 2.13 \\
\hline Hydrocynus forskalii & ND & ND & 16160 & 4.70 \\
\hline Hyperopsis bebe & ND & ND & 1295 & 0.38 \\
\hline Labeo coubie & 8214 & 1.33 & 812 & 0.24 \\
\hline Labeo senegalensis & ND & ND & 3368 & 0.99 \\
\hline Lates niloticus & ND & ND & 75428 & 21.96 \\
\hline Leptocypris niloticus & 2 & 0.00032 & 23 & 0.01 \\
\hline Malapterurus electricus & ND & ND & 1486 & 0.43 \\
\hline Marcusenus senegalensis & 13210 & 2.14 & 2049 & 0.60 \\
\hline Mormyrus macrophthalmus & ND & ND & 716 & 0.21 \\
\hline Mormyrus rume & ND & ND & 383 & 0.11 \\
\hline Oreochromis niloticus & 12504 & 2.03 & 639 & 0.19 \\
\hline Petrocephalus bane & 191 & 0.03 & 229 & 0.07 \\
\hline Petrocephalus bovei & 11290 & 1.83 & 1895 & 0.55 \\
\hline Pollimyrus isidori & 8 & 0.0012 & 57 & 0.02 \\
\hline Polypterus senegalensis & 46 & 0.01 & 27 & 0.01 \\
\hline Sarotherodon galileus & 76856 & 12.48 & 2393 & 0.70 \\
\hline Schilbe intermedius & 30332 & 4.93 & 10765 & 3.13 \\
\hline Schilbe mystus & ND & ND & 428 & 0.12 \\
\hline Synodontis eupterus & 201 & 0.03 & 95 & 0.02 \\
\hline Synodontis gambiensis & 12260 & 1.99 & 154320 & 44.93 \\
\hline Synodontis ocelifer & ND & ND & 40 & 0.01 \\
\hline Synodontis velifer & ND & ND & 27 & 0.01 \\
\hline Tilapia zillii & 14457 & 2.35 & 448 & 0.13 \\
\hline Total & 615848 & 100.00 & 343467 & 100.00 \\
\hline
\end{tabular}

$\mathrm{ND}=$ No data

In the Libga Reservoir, fish species composition in terms of weight was dominated by $S$. gambiensis $(44.93 \%)$ and L. niloticus $(21.96 \%)$. Species with percentage weight composition between 1.00 and $5.00 \%$ were $H$. niloticus $(2.13 \%)$, S. intermedius $(3.13 \%)$, H. forskalii $(4.70 \%)$, C. anguillaris $(4.96 \%)$ and $A$. occidentalis $(5.32 \%)$. Fish species which had percentage weight composition below $1.00 \%$ included $P$. isidori $(0.02 \%)$, P. bane $(0.07 \%)$, B. brachyiustus $(0.10 \%)$, M. rume $(0.11 \%), T$. zillii $(0.13 \%)$, B. leuciscus $(0.16 \%)$, O. niloticus $(0.19 \%), \quad M$. macrophthalmus $(0.21 \%)$, L. coubie (0.24\%), C. citharus $(0.25 \%)$, B. macrops $(0.29 \%)$, H. bebe $(0.38 \%)$, M. electricus $(0.43 \%), P$. bovei $(0.55 \%)$, M. senegalensis $(0.60 \%)$ and S. galileus $(0.70 \%)$.

Fish species diversity

The estimated diversity indices for the two reservoirs (Table 3) showed that the values of all the diversity indices, namely species richness (D), species diversity (H') and species evenness (J), 
were lower for the Bontanga Reservoir (1.11, 1.55 and 0.40) than for the Libga Reservoir (2.4, 2.36 and 0.52 ), respectively.

TABLE 3

Estimated diversity indices of fish species in Bontanga and Libga reservoirs in northern Ghana (July 1995 - June 1996)

$\begin{array}{lll}\text { Diversity indices } & \begin{array}{l}\text { Bontanga } \\ \text { Reservoir }\end{array} & \begin{array}{l}\text { Libga } \\ \text { Reservoir }\end{array} \\ \text { Species richness (D) } & 1.11 & 2.4 \\ \text { Species diversity (H') } & 1.55 & 2.36 \\ \text { Species evenness (J) } & 0.40 & 0.52\end{array}$

Growth parameters of important species

The most important species in the Bontanga and Libga reservoirs identified as the most frequently occurring were A. occidentalis, B. macrops, B. nurse, L. niloticus, $S$. galileus and $S$. intermedius (Table 4). The growth constant for these six species which ranged between 0.55 and 1.95 were as follows: $S$. intermedius (1.95), B. macrops (1.00), A. occidentalis (1.00), B. nurse (1.00), L. niloticus (1.00) and S. galileus (0.55). Growth performance index for the aforementioned species in the Bontanga and Libga reservoirs were as follows: L. niloticus (5.31), A. occidentalis (5.16), B. nurse (4.78), S. intermedius (4.76), S. galileus (4.65) and B. macrops (4.04).

\section{TABLE 4}

Estimated growth parameters based on length-frequency data for the major species in the Bontanga and Libga reservoirs in northern Ghana

\begin{tabular}{lcccccc} 
& \multicolumn{3}{c}{ Bontanga Reservoir } & \multicolumn{3}{c}{ Libga Reservoir } \\
Species & $L(\mathrm{~mm})$ & $K$ & $\varnothing$ & $L(\mathrm{~mm})$ & $K$ & $\varnothing$ \\
& & & & & & \\
Schilbe intermedius & 214 & 1.95 & 4.76 & & & \\
Barbus macrops & 105 & 1.0 & 4.04 & & & \\
Auchenoglanis occidentalis & 380 & 1.0 & 5.16 & & & \\
Sarotherodon galileus & 388 & 0.55 & 4.65 & & & \\
Brycinus nurse & & & & 245 & 1.0 & 5.31 \\
Lates niloticus & & & & 465 & 1.0 & 5.31
\end{tabular}

\section{Potential fish yield}

From the Morphoedaphic indices (MEI) values (Table 5), estimated potential fish yields were $86.98 \mathrm{~kg} \mathrm{ha}^{-1}$ and $97.19 \mathrm{~kg} \mathrm{ha}^{-1}$ for Bontanga and Libga reservoirs, respectively. Thus, based on their respective sizes of 770 ha and 48 ha at maximum height, an estimated wet weight yield of about $67 \mathrm{t}^{\text {year-1 }}{ }^{-1}$ and $4.7 \mathrm{t}_{\text {year }}^{-1}$ of fish could be expected from the Bontanga and Libga reservoirs, respectively.

TABLE 5

Potential fish yield based on morphoedaphic indices of Bontanga and Libga reservoirs in northern Ghana (July 1995 June 1996)

West African Journal of Applied Ecology, Vol. 12, 2008 


\begin{tabular}{|c|c|c|c|c|c|c|}
\hline \multirow[b]{2}{*}{ Months } & \multicolumn{3}{|c|}{ Bontanga } & \multicolumn{3}{|c|}{ Libga } \\
\hline & Conductivity & Depth & $M E I$ & Conductivity & Depth & $M E I$ \\
\hline Jul '95 & 87.6 & 3.7 & 23.68 & 67.6 & 1.9 & 35.58 \\
\hline Aug '95 & 69.8 & 5.2 & 13.42 & 45.7 & 3.8 & 12.03 \\
\hline Sep '95 & 64.2 & 5.8 & 11.07 & 43.0 & 3.7 & 11.62 \\
\hline Oct '95 & 67.5 & 5.8 & 11.64 & 44 & 3.8 & 11.58 \\
\hline Nov '95 & 63.4 & 5.6 & 11.32 & 48.3 & 3.4 & 14.21 \\
\hline Dec '95 & ND & ND & ND & ND & ND & ND \\
\hline ‘96 & 68.2 & 5.0 & 13.64 & 54.0 & 3.2 & 16.88 \\
\hline Feb '96 & 77.6 & 4.6 & 16.87 & 62.0 & 2.8 & 22.14 \\
\hline Mar '96 & 77.8 & 4.2 & 18.52 & 69.1 & 2.3 & 30.04 \\
\hline Apr '96 & 84.8 & 3.8 & 22.32 & 72.2 & 2.0 & 36.1 \\
\hline May '96 & 83.4 & 3.3 & 25.27 & 77.0 & 1.7 & 45.29 \\
\hline Jun '96 & 143.1 & 3.4 & 42.09 & 74.0 & 2.2 & 33.64 \\
\hline Mean 80.67 & 4.58 & 19.08 & 59.69 & 2.81 & 24.46 & \\
\hline
\end{tabular}

$* \mathrm{ND}=$ No data, Potential fish yield $(\mathrm{Y}) \mathrm{Y}=23.281 \mathrm{MEI}^{0.447}$, Bontanga $86.98 \mathrm{~kg} \mathrm{ha}^{-1}$, Libga $97.19 \mathrm{~kg} \mathrm{ha}^{-1}$.

\section{Feeding habits}

On the basis of their feeding habits, four groups (Table 6a) were identified in the Bontanga Reservoir. The carnivores (38.1\%) were dominant while the other feeding groups, namely the aufwuch-detritus and herbivores (23.24\%), semi pelagic omnivores (22.47\%) and benthic omnivores $(15.78 \%)$ followed in that order. In the Libga Reservoir, the aufwuch-detritus and herbivores $(50.1 \%)$ were dominant while the other groups, namely carnivores $(32.08 \%)$, semi pelagic omnivores $(11.78 \%)$ and benthic omnivores $(6.04 \%)$ followed in that order (Table 6b). Mochokidae (2\%), Osteoglossidae (3\%), Characidae (3\%), Clariidae (5\%), Mormyridae (4\%) and Schilbeidae (4.93\%) (Fig. 2a). In the Libga Reservoir, the dominant fish families were Mochokidae (49.97\%), Centropomidae (21.96\%), Characidae (11.7\%) and Clarotidae (5.32\%) while the fish families which constituted between $3 \%$ and $7 \%$ of the fish population were Mormyridae (2.14\%), Cyprinidae (1.54\%), Osteoglossidae (2.13\%) and Schilbeidae (3.25\%) (Fig. $2 b)$.

TABLE 6a

Forage-carnivore ratio of fishes in terms of weight in the Bontanga Reservoir in northern Ghana (July 1995 - June 1996)

Feeding type

Weight $(g)$

Percentage (\%)

Aufwuchs-detritus and herbivores

Heterotis niloticus

$18714 \quad 3.04$

Labeo coubie

$8214 \quad 1.33$

Oreochromis niloticus

125042.03

Sarotherodon galileus

76856

12.47

Synodontis eupterus

201

12260

0.03

Synodontis gambiensis

14457

1.99

Tilapia zillii

143206

2.35

Sub total

78476
3

17979 


$\begin{array}{lrr}\text { Leptocypris niloticus } & 2 & 0.01 \\ \text { Petrocephalus bane } & 191 & 0.03 \\ \text { Petrocephalus bovei } & 11290 & 1.83 \\ \text { Polypterus senegalus } & 46 & 0.01 \\ \text { Schilbe intermedius } & 30322 & 4.92 \\ \text { Sub total } & 138309 & 22.47 \\ & & \\ \text { Benthic omnivores } & & \\ \text { Auchenoglanis occidentalis } & 82681 & 13.42 \\ \text { Brienomyrus brachyistus } & 1272 & 0.21 \\ \text { Marcusenius senegalensis } & 13210 & 2.14 \\ \text { Pollimyrus isidori } & 8 & 0.01 \\ \text { Sub-total } & 97171 & 15.78 \\ & & \\ \text { Carnivores } & 223069 & 36.22 \\ \text { Clarias anguillaris } & 14093 & 2.29 \\ \text { Hemichromis fasciatus } & 237162 & 38.51 \\ \text { Sub total } & 615848 & 100.0 \\ \text { Grand total } & & \\ \text { Bontanga F/C (wt) }=\text { Total foragers (herbivores }+ \text { omnivores) } & & \\ \text { Bontanga F/C (wt) }=1.60 & \text { Carnivores } & \end{array}$

Table $6 \mathrm{~b}$

Forage-Carnivore ratio of fishes in terms of weight in the Libga Reservoir in northern Ghana (July 1995 - June 1996)

Feeding type

Weight (g)

Percentage (\%)

Aufwuchs-detritus and herbivores

Citharinus citharus

Ctenopoma kingslaye

Distichodus rostratus

Heterotis niloticus

1719

0.01

Labeo coubie

7321

0.50

Labeo senegalensis

812

2.13

Oreochromis niloticus

3368

0.24

Sarotherodon galileus

2393

0.19

Synodontis eupterus

Synodontis gambiensis

95

Synodontis ocellifer

154320

0.70

Synodontis velifer

Tilapia zillii

40

0.02

Sub total

448

0.01

0.01

0.13

172074

50.10

Semi pelagic omnivores

Alestes baremose

3000

1020

0.87

Barbus macrops

1020
552

Brycinus leuciscus

Brycinus nurse

Hyperopsis bebe

Leptocypris niloticus

20536

0.30

1295

0.16

5.98

Mormyrus macropthalmus

23

0.38

Mormyrus rume

-

Petrocephalus bane

229

0.21

Petrocephalus bovei

1895

0.11
0.07

Schilbe intermedius

10377

0.55

Schilbe mystus

428

0.12

West African Journal of Applied Ecology, Vol. 12, 2008 
Benthic omnivores

Auchenoglanis occidentalis

18290

345

2049

57

20741

Sub total

Carnivores

Clarias anguillaris

Hemichromis bimaculatus

Hemichromis fasciatus

Hydrocynus forskalii

Lates niloticus

Malapterurus electricus

Polypterus senegalus

Sub total

Grand total

$$
17037
$$

33

27

16160

75428

1486

27

110198

343467
5.32

0.10

0.60

0.02

6.04

4.96

0.01

0.01

4.70

21.96

0.43

0.01

32.08

100.0

Libga F/C (wt) $=\underline{\text { Total Foragers (Herbivores }+ \text { Omnivores) }}$

$\operatorname{Libga} \mathrm{F} / \mathrm{C}(\mathrm{wt})=2.12$

Carnivores

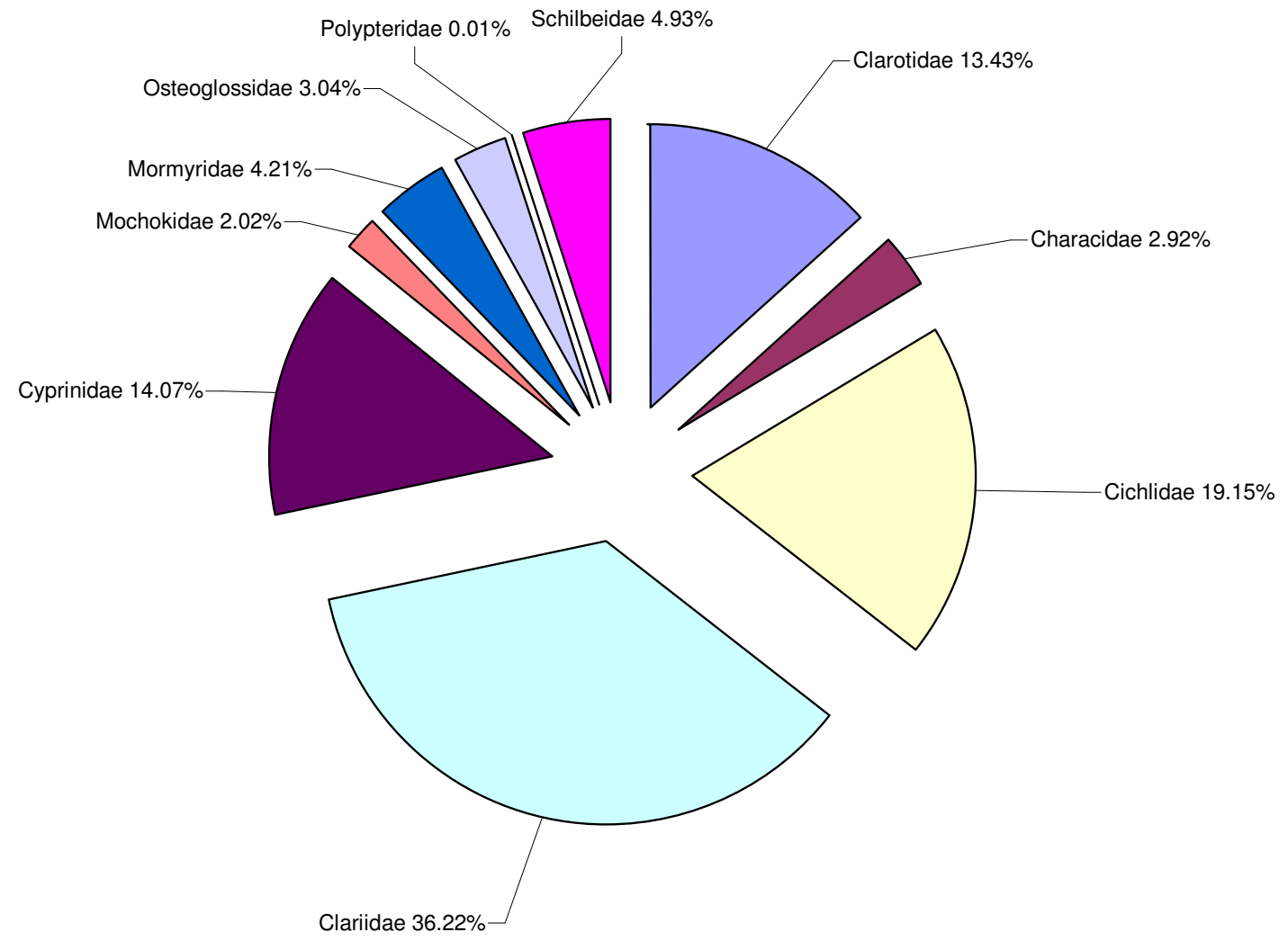

Fig. 2a. Percentage representation of fish families by weight in Bontanga Reservoir in northern Ghana 


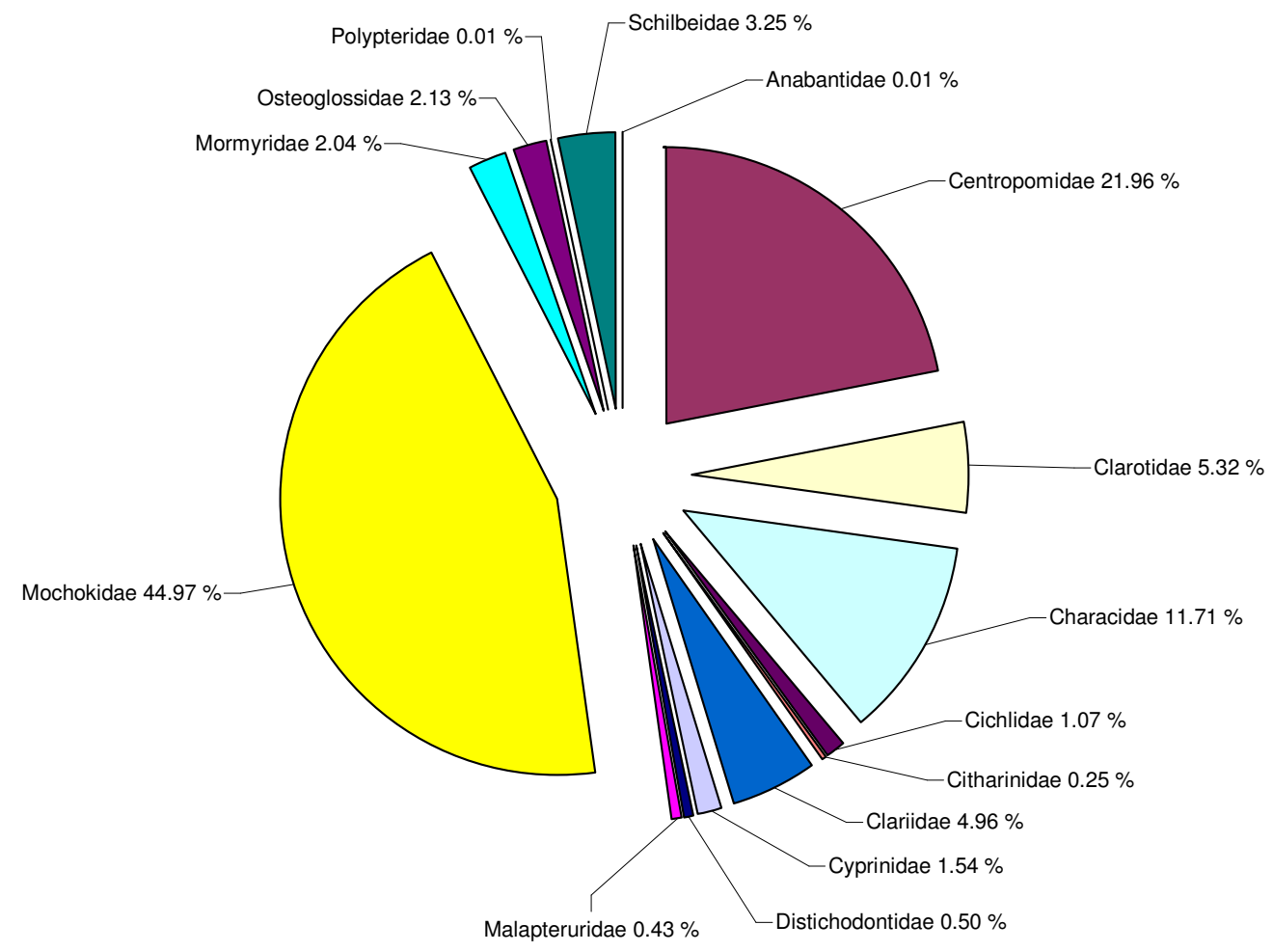

Fig. 2b. Percentage representation of fish families by weight in Libga Reservoir in northern Ghana

\section{Catch per unit effort (CPUE)}

In the Bontanga Reservoir, increasing CPUE (Fig. 3a) was related to decreasing water levels between September 1995 and January 1996 as shown by CPUE values which rose from 12,348.3 $\mathrm{g}$ by $26.2 \%$ to $16,733.6 \mathrm{~g}$ while the corresponding water levels decreased from $5.8 \mathrm{~m}$ to $5.0 \mathrm{~m}$. On the other hand, decreasing CPUE was related to decreasing water levels from January to April 1996 as shown by the fact that when CPUE dropped from 16,733.6 g to 4,751.8 g, the water levels decreased from $5 \mathrm{~m}$ to $3.4 \mathrm{~m}$.

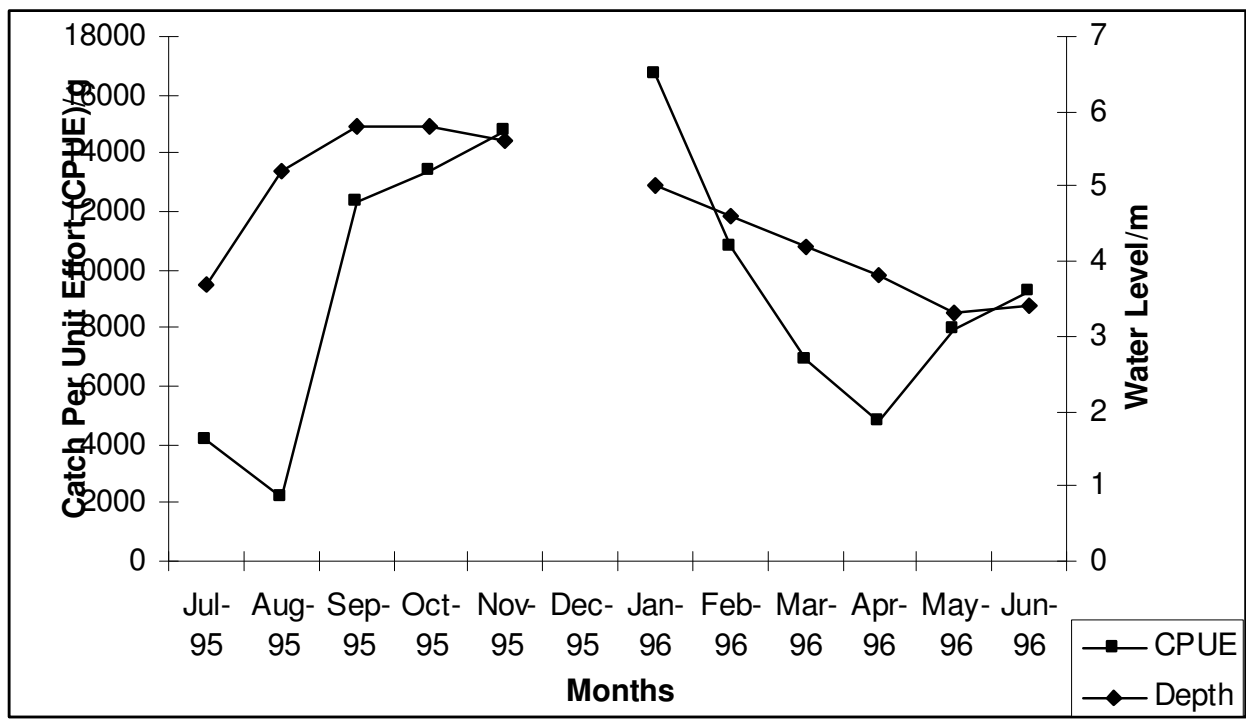

West African Journal of Applied Ecology, Vol. 12, 2008 
Fig. 3a. Monthly variation in CPUE of small mesh sized nets and water levels in Bontanga Reservoir in northern

Ghana

In the Libga Reservoir, increasing CPUE (Fig. 3b) was associated with rising but fluctuating water levels from July to December 1995. This is shown by a rise in CPUE from $556.3 \mathrm{~g}$ to $2,567.3 \mathrm{~g}$, and a rise in water level from $1.98 \mathrm{~m}$ through $3.8 \mathrm{~m}, 3.69 \mathrm{~m}, 3.84 \mathrm{~m}$ to $3.35 \mathrm{~m}$. On the other hand, fluctuating CPUE were associated with decreasing water levels from January to May 1996. This is shown by CPUE values of $1402.3 \mathrm{~g}, 2764.5 \mathrm{~g}, 14441.2 \mathrm{~g}, 2698.7 \mathrm{~g}$ and $4147.5 \mathrm{~g}$, respectively, for January to May 1996, which was associated with water level values of 3.2, 2.8, $2.44,2.0$ and $1.7 \mathrm{~m}$, respectively.

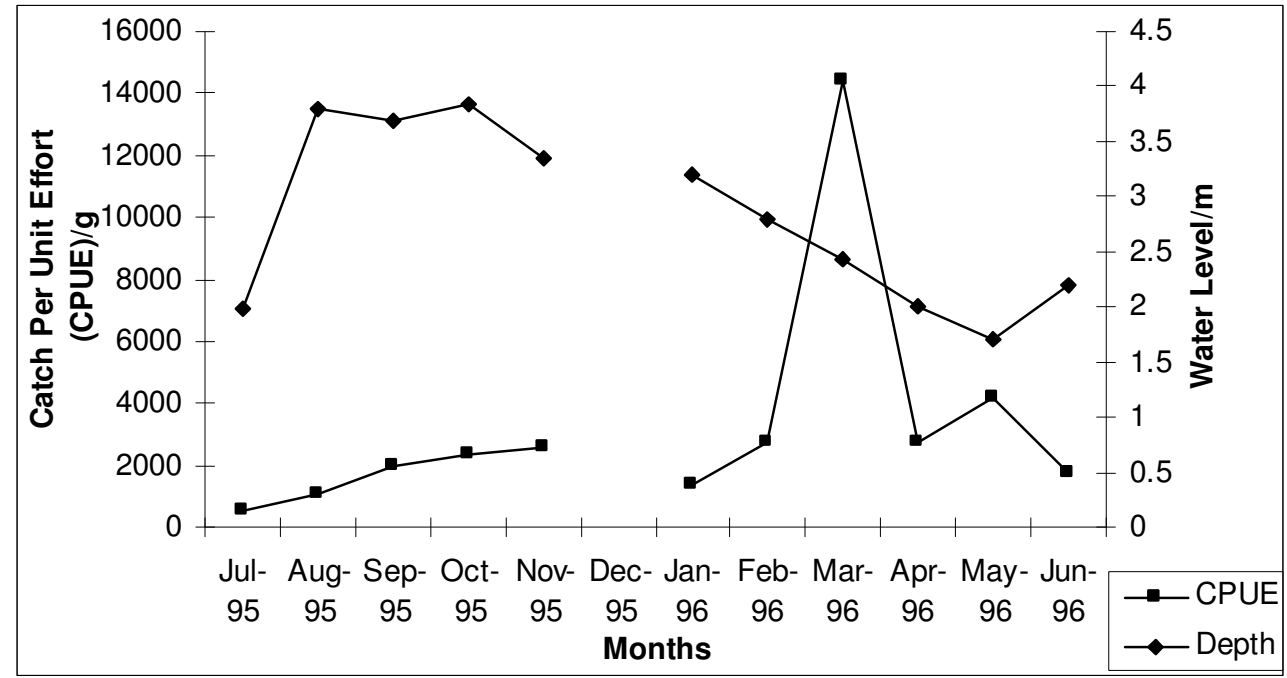

Fig. 3b. Monthly variation in CPUE of small mesh sized nets and water levels in Libga Reservoir in northern Ghana Physical parameters

The mean annual surface temperatures for the Bontanga and Libga reservoirs were $30.2{ }^{\circ} \mathrm{C}$ and $29.7{ }^{\circ} \mathrm{C}$, respectively (Table 7). There was a slight seasonal temperature variation in the case of Libga. The Bontanga and Libga reservoirs attained highest surface temperature $\left(32.0^{\circ} \mathrm{C}\right)$ in October 1995 and December 1995, respectively, while the lowest temperature $\left(26.3{ }^{\circ} \mathrm{C}\right)$ was recorded between November 1995 and February 1996. A $p \mathrm{H}$ range of 5.36-7.04 was recorded for Libga Reservoir while that for the Bontanga reservoir ranged between 5.69 and 8.67. Dissolved oxygen (DO) recorded a mean value of $15.4 \mathrm{mg} \mathrm{l}^{-1}$ in Bontanga and $16.0 \mathrm{mg} \mathrm{l}^{-1}$ in Libga with coefficient of variation of $32.2 \%$ and $48.9 \%$ R.S.D., respectively. The water at Bontanga was less turbid than that of Libga with mean values of 8.64 FTU (Formazin turbidity units) and 12.36 FTU, respectively. The annual mean conductivity value was $80.7 \mu \mathrm{S} \mathrm{cm}^{-1}$ for Bontanga and 59.7 $\mu \mathrm{S} \mathrm{cm} \mathrm{cm}^{-1}$ for Libga (Table 7). The corresponding mean total dissolved solids (TDS) values were $40.2 \mathrm{mg} \mathrm{l}^{-1}$ for Bontanga and $29.8 \mathrm{mg} \mathrm{l}^{-1}$ for Libga.

TABLE 7

Physico-chemical data on Bontanga and Libga reservoirs in northern Ghana (values are mean over sampling period July 1995-June 1996, standard deviation (SD) and \%RSD)

Parameters

Temperature $\left({ }^{\circ} \mathrm{C}\right)$

\section{Bontanga Reservoir} Annual

S.D.

30.3
$C V \%$

3.78
Libga Reservoir

Annual S.D. $\quad C V \%$

mean

$29.7 \quad 2.3$

3




$p \mathrm{H}(p \mathrm{H}$ units)
Dissolved oxygen $(\mathrm{DO})$
Biochemical oxygen demand $\left(\mathrm{BOD}_{5}\right)$
Turbidity $(\mathrm{FTU})$
Alkalinity $\left(\right.$ as $\left.\mathrm{CaCO}_{3}\right)$
Total hardness $\left(\mathrm{as} \mathrm{CaCO}_{3}\right)$
Suspended solids $(\mathrm{SS})$
Transparency $(\mathrm{m})$
Magnesium $(\mathrm{Mg})$
Calcium $(\mathrm{Ca})$
Chloride $(\mathrm{Cl})$
Phosphate $\left(\mathrm{PO}_{4}-\mathrm{P}\right)$
Sulphate $\left(\mathrm{SO}_{4}\right)$
Nitrate $\left(\mathrm{NO}_{3}-\mathrm{N}\right)$
Nitrite $\left(\mathrm{NO}_{2}-\mathrm{N}\right)$
Ammonium $\left(\mathrm{NH}_{4}-\mathrm{N}\right)$
Silicate $\left(\mathrm{SiO}_{2}\right)$
Total dissolved solids $(\mathrm{TDS})$
Conductivity $\left(\mu \mathrm{S} \mathrm{cm}^{-1}\right)$
Productivity $\left(\mathrm{g} \mathrm{C} \mathrm{m}^{-3}\right.$ day

$\begin{array}{cccccc}6.9 & - & - & 6.6 & - & - \\ 15.35 & 4.94 & 32.2 & 16.0 & 7.84 & 48.9 \\ 6.65 & 5.05 & 75.9 & 6.48 & 4.75 & 73.2 \\ 8.63 & 6.77 & 78.4 & 12.4 & 15.47 & 125 \\ 39.9 & 16.6 & 41.5 & 29.3 & 11.1 & 37.9 \\ 18.5 & 9.41 & 50.9 & 13.4 & 6.27 & 46.9 \\ 7.73 & 5.96 & 77.2 & 10.9 & 8.31 & 76.2 \\ 0.597 & 0.203 & 34.0 & 0.541 & 0.302 & 55.9 \\ 0.915 & 1.18 & 129 & 0.428 & 0.59 & 139 \\ 7.12 & 2.82 & 39.6 & 5.70 & 2.08 & 36.4 \\ 13.14 & 3.41 & 25.9 & 13.1 & 2.58 & 19.8 \\ 0.044 & 0.053 & 110 & 0.116 & 0.187 & 162 \\ 1.45 & 1.81 & 125 & 3.77 & 5.75 & 152.3 \\ 0.350 & 0.436 & 125 & 0.721 & 0.875 & 121.3 \\ 0.015 & 0.022 & 153 & 0.066 & 0.126 & 190 \\ 0.313 & 0.175 & 55.8 & 0.259 & 0.182 & 70.5 \\ 16.4 & 7.0 & 42.6 & 10.4 & 3.65 & 35.1 \\ 40.2 & 11.2 & 27.8 & 29.8 & 6.52 & 21.9 \\ 80.7 & 22.4 & 27.7 & 59.7 & 13.0 & 21.8 \\ 3.73 & 3.58 & 96.1 & 5.05 & 5.59 & 111\end{array}$

$N B$ : All results are in $\mathrm{mg}^{-1}$ except $p \mathrm{H}$ and where otherwise stated.

\section{Nutrients}

Only traces of phosphate $\left(\mathrm{PO}_{4}-\mathrm{P}\right)$, nitrates $\left(\mathrm{NO}_{3}-\mathrm{N}\right)$ and nitrites $\left(\mathrm{NO}_{2}-\mathrm{N}\right)$ were recorded in the Bontanga Reservoir while the corresponding concentrations in the Libga Reservoir were 0.116

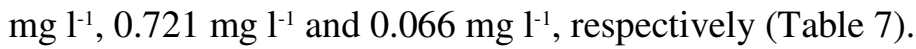

\section{Productivity}

The mean productivity for Bontanga Reservoir was $3.73 \mathrm{~g} \mathrm{C} \mathrm{m}^{-3}$ day $^{-1}$ and the corresponding concentration for Libga Reservoir was $5.05 \mathrm{~g} \mathrm{C} \mathrm{m}^{-3}$ day $^{-1}$ (Table 7).

\section{Discussion}

The study area falls within the typical Guinea savanna zone where water levels decrease greatly during the long dry season (October-June) mainly through evapotranspiration. The lack of preimpoundment data on both reservoirs makes it difficult for comparison and comprehensive comments to be made especially regarding the formative stages of the two reservoirs.

Studies on existing species composition and state of balance between fish populations in a water body are essential in predicting the possibility or otherwise of the populations yielding annual harvestable crops (Swingle, 1950). Such predictions provide the necessary basis for improved management of impoundments (Tang, 1970). The current fish composition of the reservoirs could be regarded as having survived the combined effects of the long dry periods and changes associated with the formation and/or stabilization phases of the reservoirs. The number of fish species in the Bontanga Reservoir was lower than that of the Libga Reservoir.

The higher fish species richness for the Libga Reservoir was anticipated since the reservoir is located within the floodplain of the White Volta and indications are that the reservoir gets stocked by fish from the annual flooding of the main river and inflow waters associated with the reservoir. The composition of species in the Bontanga Reservoir during the study period was similar to that of the Dawhenya Reservoir a decade after creation (Blay Jr, 1985). Indications are that there had been the development of fish populations dominated by fish-eating species over the years in the Libga Reservoir which could, therefore, be regarded as an important cove in the floodplains of the White Volta into which predators especially Lates niloticus found their way. 
The most important feeding group in the Bontanga Reservoir was the aufwuch-detritus and herbivores while in the Libga Reservoir, the major feeding group was the carnivores, especially Lates niloticus which constituted about $22 \%$ of ichthyomass studied. The estimated F/C ratios of 1.60 and 2.12, respectively, for Bontanga and Libga reservoirs fell within the acceptable range of 1.4-10.0 for balanced fish populations (Blay Jr, 1985; Ofori-Danson et al., 1993; Ofori-Danson \& Antwi, 1994), indicating a balanced fish community status and a suitable ecological balance between carnivorous fishes and their prey.

In the Bontanga Reservoir, Datua (1989) recorded 10 species of commercial importance. Those species were also observed in the current study as well as in the catches of fishermen operating in the reservoir. The tilapias (especially S. galileus) formed the mainstay of the fishery in the Bontanga Reservoir due to food availability in the reservoir and successful reproduction. The prominence of Heterotis niloticus in the catches of fishermen confirmed the assertion of Mayachi (1991) that the species had assumed a commercially important status in the Bontanga Reservoir. The limited number obtained during the sampling period could probably be due to factors related to the use of prohibited fishing gear which could have constituted a devastating pressure on the species.

Barbus macrops, the major species encountered during the study, was conspicuously absent from commercial catches of fishers in the Bontanga Reservoir. The situation could be attributed to the regulatory measures being enforced in the reservoir. It was apparent that the relatively large mesh-sized gill nets permitted in the reservoir were in common use by the fishermen. These nets were clearly selecting the bigger-sized fishes. In the Libga Reservoir, the species composition seemed to rise with the floods so it is important that any enhancement strategy for the reservoir takes cognizance of this annual influx of species with the floods.

In this study, rising CPUE of the small mesh-sized nets which is associated with rising water (July-September) and high water (October-December) periods of the Bontanga Reservoir could be attributed partly to increased vulnerability of fish fingerlings to netting and partly to high success of reproduction leading to increased numbers of semi-pelagic species which are also vulnerable to netting. On the other hand, the relatively low CPUE of smaller mesh-sized nets associated with high waters in the Libga Reservoir, though comparable to the results expressed by Amarasinghe \& Pitcher (1986), Amarasinghe (1987) and Blay Jr. \& Asabere-Ameyaw (1993) could be attri-buted to less success of reproduction and the generally poor representation of semipelagic fishes in the reservoir. No clear seasonality patterns could be observed for CPUE in both reservoirs.

Estimates of potential fish yields of $86.98 \mathrm{~kg} \mathrm{ha}^{-1}$ and $97.17 \mathrm{~kg} \mathrm{ha}^{-1}$ for Bontanga and Libga reservoirs, respectively, from this study were comparable to those of much smaller reservoirs such as Busunu (82.62 kg ha-1), Achubunyo (75.05 kg ha-1) and Mahama (90.19 kg ha-1) which are located in the same geoclimatic area of the Northern Region of Ghana (Abban et al., 1994). Vanden Bossche \& Bernacsek (1990) estimated mean potential yields of $450 \mathrm{~kg} \mathrm{ha}^{-1}$ and $167 \mathrm{~kg}$ $\mathrm{ha}^{-1}$ for two Tanzanian reservoirs, namely Ngwazi $\left(5.1 \mathrm{~km}^{2}\right)$ and Malya $\left(0.7 \mathrm{~km}^{2}\right)$. Comparing Ngwazi $\left(5.1 \mathrm{~km}^{2}\right)$ and Malya $\left(0.7 \mathrm{~km}^{2}\right)$ with Bontanga $\left(7.7 \mathrm{~km}^{2}\right)$ and Libga $\left(0.48 \mathrm{~km}^{2}\right)$ that are of similar sizes, it is apparent that the yields in the Bontanga and Libga reservoirs are considerably low probably due to differences in ionic content. The scope for improvement of yields in the Bontanga and Libga reservoirs would require sound environ-mental, scientific and social measures such as the institution of regular monitoring, stocking with indigenous species, management of habitat to prevent sedimentation as well as clearing of vegetative cover, regula-tion of water levels, fishing regulations and control, and education of riparian communi-ties, among others.

The reservoir waters were slightly acidic (6.4-6.9) from June to October and the two reservoirs were well oxygenated (15.4 $\mathrm{mg} \mathrm{l}^{-1}$ and $16.0 \mathrm{mg} \mathrm{l}^{-1}$ for Bontanga and Libga, respectively), with

West African Journal of Applied Ecology, Vol. 12, 2008 
relatively high oxygen demanding substances (mean BOD of $6.65 \pm 5.05$ for Bontanga and $6.48 \pm$ 7.4 for Libga). There was variation in BOD levels in Libga, reflecting diffuse sources of oxygen demanding substances. There was less variation in dissolved oxygen (DO) levels in Bontanga Reservoir than in the Libga Reservoir as evidenced by the coefficient of variation values. This was due to the fact that Libga Reservoir had more algae resulting in increased photosynthetic activities. After sunset, DO concentrations decline as photosynthesis stops and all plants and animals in the reservoir consume oxygen. The high levels of dissolved oxygen recorded in these reservoirs are indications of the super-saturated nature of the oxygen present in the water. Most DO in reservoirs are produced during photosynthesis by aquatic plants including algae. These pieces of evidence were confirmed with productivity measurements. A concentration of $5 \mathrm{mg} \mathrm{l}^{-1}$ DO is suitable for optimum fish growth and health (Hart, 1974). The wide $p \mathrm{H}$ range for Bontanga Reservoir, with a difference of about 10 units, was mainly due to the presence of algae in the reservoir. Generally, reservoir $p \mathrm{H}$ varies throughout the day due to respiration and photosynthesis.

The striking feature of the chemistry of Bontanga Reservoir was its high ionic content as reflected in the high conductivity and total dissolved solids (TDS) values recorded. The variation of turbidity over the entire sampling period was lower for Bontanga $(78.4 \%)$ than for Libga $(125 \%)$, reflecting the type of soil and the nature of the terrain in their catchment areas. The ionic content could be attributed to two factors, namely the advanced weathering of rocks and soils in the Bontanga Reservoir and the low solubility of the underlying rocks, namely granite and chists found in the Libga catchment area. The relatively higher nutrient levels in Libga Reservoir could be due to the washing of phosphate and nitrates from the nearby agricultural farms into the reservoir. This also confirms the variation in BOD levels in the Libga Reservoir.

Generally, high productivity was observed for both reservoirs during the rainy season with the Libga Reservoir slightly higher due to higher algal production. In the dry season, however, productivity was generally low with the higher productivity being observed in Bontanga Reservoir. Changes in productivity of both reservoirs can be explained by the photosynthetic activities of algae. This also shows that the nutrient level of the Libga Reservoir was slightly higher than that of the Bontanga Reservoir.

\section{Conclusion}

In the Bontanga Reservoir, 21 species belonging to 18 genera and 10 families were encountered. Four families, namely Clariidae, Cichlidae, Cyprinidae and Clarotidae constituted almost $83 \%$ of the fishery in the Bontanga Reservoir with the major species being $C$. anguillaris, A. occidentalis, B. macrops and S. galileus. In the Libga Reservoir, 36 species representing 27 genera and 15 families were observed. Two families, namely Mochokidae and Centropomidae constituted almost $67 \%$ of the fishery resources in the Libga Reservoir with S. gambiensis and the Nile perch, L. niloticus, being the dominant species.

The Bontanga Reservoir had lower fish species richness than the Libga Reservoir as expected due to the location of the latter within the flood plains of the White Volta, where it gets stocked with fish from the annual flooding of the main river as well as from inflow waters associated with the reservoir. The most important feeding group in the Bontanga Reservoir was the carnivores. In the Libga Reservoir, the major feeding group was the aufwuch-detritus and herbivores. Estimates of forage-carnivore ratio gave indications of a suitable ecological balance between foragers and carnivores in both the Bontanga and Libga reservoirs.

The estimated annual harvestable fish from Bontanga and Libga reservoirs were comparable with that for reservoirs of smaller sizes in the same geoclimatic area in the Northern Region while yields were lower in comparison with reservoirs of similar sizes in different geoclimates. Some interventions that may be considered to improve the potential fish yield of both reservoirs include regular monitoring programmes, stocking with indigenous species, management of habitat to 
prevent sedimentation, clearing of vegetative cover, regulation of water levels, fishing regulations and control, and education of riparian communities, among others. It is important to note that strategies to be adopted towards the management of fisheries in reservoirs would depend on their individual productivity as well as on social and economic factors, such as the presence or absence of fishers (Marshall \& Maes, 1995).

Generally, both reservoirs were more productive in the rainy season than in the dry season, with Libga slightly higher in the rainy season and Bontanga slightly higher in the dry season. Both reservoir waters were slightly acidic from June to October and well oxygenated with relatively high oxygen demanding substances. Variations in BOD levels in Libga reflect diffuse sources of oxygen demanding substances. There was less variation in DO levels in Bontanga Reservoir than in Libga Reservoir as evidenced by the coefficient of variation values. The Bontanga Reservoir showed strikingly higher ionic content attributed to advanced weathering of rocks and soils than Libga Reservoir attributed to low solubility of underlying rocks. Nutrient levels were lower in Bontanga Reservoir than in Libga Reservoir.

\section{Acknowledgement}

Support given by Dr J. Samman and the entire staff of the Tamale Field Station of the CSIRWater Research Institute is gratefully acknowledged. Also acknow-ledged are Dr E. K. Abban and Dr P. K. Ofori-Danson for their criticism and advice. The field and laboratory assistance of Messrs G. B. Amegbe, Obed Tonlaar and Humphrey F. Darko during the study period is acknowledged. The use of CSIR-Water Research Institute facilities is also gratefully acknowledged.

\section{References}

Abban E. K., Ofori-Danson, P. K. and Amevenku F. K. Y. (1994). Fish and fisheries of reservoirs as index of fishery and aquaculture potential of reservoirs. In Fisheries and aquaculture development assessment of impoundments in West Gonja District, Northern Ghana. (E. K. Abban, P. K. Ofori-Danson and C. A. Biney, ed.). Institute of Aquatic Biology Technical Report No. 136.

Amarasinghe U. S. (1987). Status of the fishery of Pimburettewa Wewe, a man-made lake in Sri Lanka. Aquacult. Fish. Mgmt 18: 375-385.

Amarasinghe U. S. and Pitcher T. J. (1986). Assessment of Fishing Effort in Parakram Samudra, an Ancient manmade Lake in Sri Lanka. Fish Res. 4: 271-282.

APHA-AWWA-WPCF (1998). Standard Methods for the Examination of Water and Wastewater. 20th edn. pp. 172978. APHA-AWWA-WPCF, Washington DC.

Blay J. Jr (1985). Observations on the balance in fish populations in a small reservoir in Ghana. Fish. Res. 3: 1-11.

Blay J. Jr and Asabere-Ameyaw A. (1993). Assessment of the fishery of a stunted population of the cichlid, Sarotherodon melanotheron, in a closed lagoon in Ghana. J. appl. Ichthyol. 9: 1-11.

Byrd I. B. and Moss D. D. (1957). The production and management of Alabama's state-owned public fishing lakes. Trans Am. Fish. Soc. 85: 208-216.

Davies W. D. (1973). Managing Small Impoundments and Community Lakes. In Proceedings of 27th Annual Conference of Southeastern Association of Game and Fisheries Commissioners, USA. pp. 347-355.

Datua E. B. (1989). The Fishes and Fisheries of Bontanga Irrigation Dam in Tamale, Northern Ghana. (Dip. Thesis.), Kwame Nkrumah University of Science \& Technology, Kumasi, Ghana. 69 pp.

deGraft-Johnson K. A. A. (1995). A survey of Aquatic Weeds in Ghanaian Waterbodies - Ecowas Floating Weeds Project. A Report for Euroconsult of Netherlands/Transcon Ltd. of Nigeria.

Gayanilo F. C. Jr, Sparre P. and Pauly D. (1996). The FAO-ICLARM Stock Assessment Tool (FiSAT) User's Guide. FAO Computerized Info. Ser. (Fish) No 7. 126 pp.

Hart B. T. (1974). A Compilation of Australian Water Quality Criteria. Australian Water Resources Council Technical Paper No. 7.

Irrigation Development Authority (IDA) (1986). Progress Report on Bontanga Irrigation Project.

Lévêque C., Fairhurst C. P., Abban E. K., Paugy D., Curtis M. S. and Traore K. (1988). Onchocerciases Control Programme in West Africa. Ten Year Monitoring of Fish Populations. Chemosphere 17: 421-440.

Lévêque C., Pauly D. and Teugels G. G. (ed.) (1992). Faune des poisons d'eaux et saumatre de l'Afrique de l'ouest (TOME2). ORSTOM et MRAC, Paris. 902 pp.

West African Journal of Applied Ecology, Vol. 12, 2008 
Margalef R. (1968). Perspectives in Ecological Theory. University of Chicago Press, Chicago. 111 pp.

Marshall B. E. (1984). Small Pelagic Fishes and Fisheries in African Inland Waters. CIFA Technical Paper No. 29. 68 pp.

Marshall B. E. and Maes M. (1995). Small Water Bodies and their Fisheries in Southern Africa. CIFA Technical Paper No. 29. 68 pp.

Mayachi S. Z. (1991). Aquaculture in water resources development: A case study of the Bontanga Irrigation Project. (Diploma Thesis.) Kwame Nkrumah University of Science and Technology, Kumasi, Ghana.

Ofori-Danson P. K., Abban E. K. and Amevenku F. K. Y. (1993). Status of Fish and Fishery in the Densu-Weija Reservoir Fourteen Years after Impoundment. Institute of Aquatic Biology Technical Report 128. CSIR-Water Research Institute, Accra, Ghana

Ofori-Danson P. K. and Antwi L. A. K. (1994). Limnology of a Tropical Reservoir (The Kpong Reservoir in Ghana). Trop. Ecol. 34 (1): 75-87.

Pielou E. C. (1969). An Introduction to Mathematical Ecology. Jonn Wiley and Sons, New York.

Ryder R. A., Kerr S. R., Loftus K. H. and Regier H. A. (1974). The morphoedaphic index, a fish yield estimator review and evaluation. J. Fish. Res. Bd Can. 31: 663-688.

Shannon E. C. and Wiener W. (1963). The Mathematical Theory of Communication. University of Illinois Press, Urbana, $117 \mathrm{pp}$.

Swingle H. S. (1950). Relationships and Dynamics of Balanced and Unbalanced Fish Populations. Alabama Polytechnic Inst. Agric. Exp. Stn. Bull. No. 274. 74 pp.

Tang Y. A. (1970). Evaluation of Balance between Fishes and Available Fish Foods in Multispecies Fish Culture Ponds in Taiwan. Trans Am. Fish. Soc. 99: 708-718.

Vanden Bossche J.-P and Bernacsek G. M. (1990). Source Book for the Inland Fishery Resources of Africa, Vol. 1. CIFA Technical Paper No. 18/1. 240 pp. 